\title{
Doença de Chagas no Estado de São Paulo: comparação entre pesquisa ativa de triatomíneos em domicílios e notificação de sua presença pela população em área sob vigilância entomológica
}

\author{
Chagas' disease in São Paulo State: a comparative study between systematic \\ search and inhabitants' report of bugs in the area under \\ entomological surveillance
}

\author{
Rubens Antonio Silva, Paula Regina Bonifácio e Dalva Marli Valério Wanderley
}

\begin{abstract}
Resumo A vigilância entomológica da doença de Chagas no Estado de São Paulo tem adotado um esquema que considera a localidade como unidade de trabalho e prioriza as ações de acordo com níveis de infestação obtidos a partir de buscas sistemáticas de triatomíneos (rotina) na área mais infestada e nas residências de moradores que notificam presença desses insetos (atendimento a notificação). As espécies triatomínicas de maior presença no Estado atualmente são Triatoma sordida e Panstrongylus megistus. Um estudo comparativo dos índices de infestação obtidos para casas e peridomicílios em pesquisas de rotina e atendimento à notificação nos biênios 90/91, 92/93 e 94/95, apontou percentuais médios de positividade para intradomicílio de 1,3 na rotina e 6,2 no atendimento. Para o peridomicílio os valores foram de 8,6 e 18,2, respectivamente, sem diferença entre os biênios. Os atendimentos às notificações demonstraram percentuais médios de positividade (encontro de foco) em $26 \%$ das casas. Foi constatado ainda que as notificações procederam de localidades com níveis de infestação iniciais nulos $(I=0)$, intermediários $(I<5)$ e elevados $(I>5)$, não obstante os percentuais de atendimentos positivos terem sido maiores naquelas cujos níveis de infestacão iniciais eram elevados. Estes dados reforçam a importância da notificação na vigilância de espécies vetoras que se caracterizam pelo caráter invasivo, como aquelas presentes atualmente no Estado de São Paulo.
\end{abstract}

Palavras-chaves: Doença de Chagas. Triatomíneos. Vetores. Vigilância entomológica.

\begin{abstract}
Entomological surveillance of Chagas' disease in São Paulo State has adopted a scheme that uses the locality as a unit for field work. Actions are prioritized according to infestation levels obtained by systematic search of triatomine (routine actions), bugs in areas with high infestation levels and in houses whose inhabitants report the presence of the bugs (reply to notification). The most prevalent triatominic species in Sao Paulo State at present are Triatoma sordida and Panstrongylus megistus. A comparative study of infestation levels obtained in houses and surroundings in routine search and replies to notification in the two-year periods of 90/91, 92/93 and 94/95, has shown average percentage positivity of 1.3 indoors in routine activity and 6.2 in the reply to notification. For the house surroundings the values were 8.6 and 18.2 respectively, no differences being found between the twoyear periods. Replies to notification showed average percentage positivity (focus) in $26 \%$ of the houses. It was verified that the notifications had come from localities with prior infestation levels equal to zero $(I=O)$, intermediary infestation levels $(I<5)$ and high infestation levels $(I>5)$, although the percentages of positive replies to notification were higher in those localities with high prior infestation levels. These results reinforce the importance of notification in the surveillance of vector species of the invasive type like those currently prevalent in Sao Paulo State.
\end{abstract}

Key-word: Chagas' disease. Triatomine. Vectors. Entomological Surveillance.

Superintendência de Controle de Endemias, São Paulo, SP.

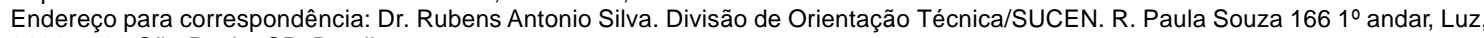
01027-000 São Paulo, SP, Brasil.

Fax: $5511229-8292$

E-mail rubens@sucen.sp.gov.br

Recebido para publicação em 13/7/98. 
As ações sistemáticas de combate aos vetores da doença de Chagas por meio do uso de inseticidas nos domicílios da zona rural paulista, iniciados em 1950, lograram êxito no sentido de interromper a transmissão natural da enfermidade causada por Triatoma infestans, já na década de $70^{8}$. Com a eliminação do Triatoma infestans o serviço de controle passou a desenvolver uma estratégia de vigilância sobre as espécies secundárias ${ }^{10}$. Esta estratégia determinou pesquisa triatomínica em áreas de infestação residual e participação da população na detecção de insetos invasores em seus domicílios ${ }^{9}$. Ao longo do tempo pode-se constatar que o Programa de Controle se organizou de acordo com a situação epidemiológica, permitindo que fossem incorporadas readequações racionalizadoras de atividades ${ }^{12}$.

As normas operacionais do Programa, a partir de 1985, passaram a considerar a localidade como unidade de programação para pesquisas triatomínicas de rotina. Este trabalho, em todas as localidades rurais, foi desenvolvido com o objetivo de identificar e combater focos residuais de vetores existentes nos peridomicílios, visto que as espécies presentes eram predominantemente peridomiciliares. A espécie triatomínica com maior presença no estado nos últimos anos é o Triatoma sordida, seguida por Panstrongylus megistus e Rhodinus neglectus. As localidades tem sido classificadas, de acordo com níveis de infestação, em prioridades que determinam a periodicidade das atividades de campo a serem cumpridas. Após cinco anos de trabalho nas localidades rurais, foram introduzidas modificações na vigilância com direcionamento das atividades de pesquisa triatomínica para áreas infestadas. Além disso, independentemente do nível de infestação das localidades, são atendidas todas as notificações de triatomíneos encaminhadas por moradores. Quando esta pesquisa é positiva o domicílio é submetido a tratamento químico. O trabalho de pesquisa triatomínica e tratamento químico nas áreas infestadas é desenvolvido ao longo de um período de dois anos. Ao fim de cada biênio, os resultados obtidos nas pesquisas, traduzidos em índices de infestação de localidades, irão determinar a nova situação epidemiológica e, consequentemente, o planejamento das ações.

O presente trabalho objetiva realizar uma avaliação das acões de pesquisa triatomínica desenvolvidas após a introdução dessas modificações como forma de subsidiar a vigilância.

\section{MATERIAL E MÉTODOS}

Foram analisados os resultados obtidos no Programa de Controle da doença de Chagas no Estado de São Paulo desenvolvido pela Superintendência de Controle de Endemias (SUCEN), para os biênios 1990/91, 1992/93, 1994/95, a partir de modificações introduzidas nas ações de vigilância. A partir de 1990 as ações de vigilância têm preconizado atividades de rotina de pesquisa de triatomíneos em localidades por meio de capturas realizadas pelas equipes de campo da SUCEN ou o atendimento às notificações de vetores encaminhados pela população. Baseando-se nos percentuais fixados por Silva e cols ${ }^{8}$, quando da introdução do critério de prioridades, as localidades foram classificadas em Prioridade 1 quando a infestação intradomiciliar (II) fosse maior ou igual a 5 e/ou peridomiciliar (IP) maior ou igual a 10 (infestação alta). Nestas tem sido realizadas pesquisas sistemáticas de rotina com periodicidade bienal. Na Prioridade 2 , foram incluídas as localidades com II entre zero e 5 e/ou IP entre zero e 10 (infestação intermediária) nas quais são realizadas pesquisas em uma amostra de localidades a cada biênio. Em Prioridade 3 foram classificadas as localidades com II e IP iguais a zero. Em todas as localidades, independentemente da prioridade em que estão classificadas, tem sido desenvolvidas atividades de atendimento às notificações de triatomíneos encaminhadas pelos moradores. Ao final de dois anos de trabalho, os resultados alcançados traduzidos em índices de infestação de localidades obtidos na atividade de rotina $(\mathrm{II}=$ casas positivas/casas pesquisadas $\mathrm{X} 100$ ou IP = peridomicílios positivos/peri pesquisados $X 100$ ) e no atendimento às notificações ( $\mathrm{II}=$ atendimentos positivos no intradomicílio/ número de casas existentes X 100 ou IP = atendimentos positivos no peridomicílio/ número de casas existentes X 100), irão determinar a nova classificação das mesmas e, consequentemente, a programação do trabalho para o próximo biênio.Neste trabalho foram comparados os resultados de positividade de casas e peridomicílios obtidos nas atividades de rotina de pesquisa de triatomíneos com a atividade de notificação desencadeada por parte dos moradores. 


\section{RESULTADOS}

No período de 1990 à 1995, foram atendidas pelas equipes de campo da SUCEN 13.369 notificações de triatomíneos encaminhadas pela população onde em 3.510 casas $(26,2 \%)$ foi constatada a presença de focos. Estes focos foram encontrados no intradomicílio $(6,2 \%)$, peridomicílio $(18,2 \%)$ e no intra e peridomicílio simultaneamente $(1,8 \%)$ (Tabela 1). Observou-se queda no número absoluto de notificações atendidas ao longo do período e manutenção da positividade.

Tabela 1 - Notificações de triatomíneos atendidas e resultados dos atendimentos segundo o local de coleta. Estado de São Paulo, 1990 à 1995.

\begin{tabular}{|c|c|c|c|c|c|c|c|c|c|}
\hline \multirow{4}{*}{ Ano } & \multirow{4}{*}{$\begin{array}{c}\text { Notificações } \\
\text { atendidas }\end{array}$} & \multicolumn{8}{|c|}{ Resultado dos atendimentos } \\
\hline & & & & & & pos & vas & & \\
\hline & & \multicolumn{2}{|c|}{ negativas } & \multicolumn{2}{|c|}{ intradomicílio } & \multicolumn{2}{|c|}{ peridomicílio } & \multicolumn{2}{|c|}{ ambos } \\
\hline & & № & $\%$ & № & $\%$ & № & $\%$ & № & $\%$ \\
\hline 1990 & 2868 & 2158 & 75,2 & 159 & 5,5 & 508 & 17,8 & 43 & 1,5 \\
\hline 1991 & 2567 & 1951 & 76,0 & 160 & 6,2 & 426 & 16,6 & 30 & 1,2 \\
\hline 1992 & 2536 & 1808 & 71,3 & 155 & 6,1 & 535 & 21,1 & 38 & 1,5 \\
\hline 1993 & 2071 & 1574 & 76,0 & 126 & 6,1 & 327 & 15,8 & 44 & 2,1 \\
\hline 1994 & 2298 & 1576 & 68,6 & 165 & 7,2 & 474 & 20,6 & 83 & 3,6 \\
\hline 1995 & 1029 & 792 & 76,9 & 64 & 6,2 & 169 & 16,5 & 04 & 0,4 \\
\hline Total & 13369 & 9859 & 73,8 & 829 & 6.2 & 2439 & 18,2 & 242 & 1,8 \\
\hline
\end{tabular}

Fonte: SUCEN

As notificações de triatomíneos procederam de localidades com infestação alta (P1), intermediária (P2) e nula (P3). O número médio de notificações por localidade foi de 1,8 . Em números absolutos constatou-se mais notificações em localidades com infestação nula seguidas de localidades com infestação intermediária e alta. No entanto, os percentuais de positividade obtidos seguiram a ordem inversa e o número absoluto de atendimentos positivos, segundo prioridade, apresentou comportamento descendente nos três biênios $(p<0,05)$ (Tabela 2).

Tabela 2 - Localidades com notificação de triatomíneos e resultados dos atendimentos às notificações segundo prioridades. Estado de São Paulo, biênios 1990/91, 1992/93 e 1994/95.

\begin{tabular}{|c|c|c|c|c|c|c|c|c|c|c|c|c|c|c|c|}
\hline \multirow[b]{2}{*}{ Biênio } & \multicolumn{5}{|c|}{ P 1} & \multicolumn{5}{|c|}{ P 2} & \multicolumn{5}{|c|}{ P 3} \\
\hline & $\begin{array}{l}\text { Loc } \\
\text { exist }\end{array}$ & $\begin{array}{l}\text { Loc } \\
\text { atend }\end{array}$ & $\begin{array}{c}\text { № atend } \\
\text { loc }\end{array}$ & atend & $\%$ posit * & $\begin{array}{l}\text { Loc } \\
\text { exist }\end{array}$ & $\begin{array}{l}\text { Loc } \\
\text { atend }\end{array}$ & $\begin{array}{c}\text { № Atend } \\
\text { loc }\end{array}$ & atend & $\%$ posit * & $\begin{array}{l}\text { Loc } \\
\text { exist }\end{array}$ & $\begin{array}{l}\text { Loc } \\
\text { atend }\end{array}$ & $\begin{array}{c}\text { № Atend } \\
\text { loc }\end{array}$ & atend & $\%$ posit * \\
\hline $1990 / 91$ & 776 & 277 & 1,9 & 233 & 84,1 & 1371 & 776 & 1,8 & 601 & 77,4 & 12732 & 1825 & 1,9 & 595 & 32,6 \\
\hline $1992 / 93$ & 613 & 358 & 1,6 & 245 & 68,4 & 1780 & 977 & 2,0 & 726 & 74,3 & 12158 & 1163 & 1,6 & 98 & 8,4 \\
\hline $1994 / 95$ & 512 & 173 & 1,9 & 141 & 81,5 & 2052 & 469 & 1,7 & 300 & 63,9 & 11360 & 736 & 1,6 & 277 & 37,6 \\
\hline
\end{tabular}

Fonte: SUCEN

* \% calculado sobre o número de localidades com atendimento

Quando comparamos as atividades de rotina e atendimento à notificação no período estudado, pode-se constatar que o atendimento à notificação demonstrou maior eficácia na detecção de focos tanto para o intradomicílio (casas) como para o peridomicílio. Os percentuais médios de positividade obtidos no atendimento foram de 6,2 para o intra e 18,2 para o peridomicílio, enquanto que a rotina demonstrou percentuais de 1,3 e 8,6 para o intra e peridomicílio, respectivamente (Tabela 3 ). Foi observado aumento nos percentuais de positividade obtidos para intra e peridomicílio na rotina. No atendimento à notificação observouse manutenção da positividade no intradomicílio e aumento no peridomicílio $(p<0,05)$. 
Tabela 3 - Casas e Peridomicilios pesquisados e positivos em atividade de rotina e atendimento à notificação de triatomineos - Estado de São Paulo, biênios 1990/91, 1992/93 e 1994/95.

\begin{tabular}{|c|c|c|c|c|c|c|c|c|c|c|c|c|}
\hline \multirow{3}{*}{ Biênio } & \multicolumn{6}{|c|}{ Rotina } & \multicolumn{6}{|c|}{ Atendimento à notificação } \\
\hline & casas & casas & & peri & peri & & casas & casas & & peri & peri & \\
\hline & pesq & posit & $\%$ & pesq & posit & $\%$ & pesq & posit & $\%$ & pesq & posit & $\%$ \\
\hline 1990/91 & 25604 & 219 & 0,9 & 22484 & 1640 & 7,3 & 5435 & 319 & 5,9 & 5435 & 934 & 17,2 \\
\hline $1992 / 93$ & 43036 & 479 & 1,1 & 38355 & 3122 & 8,1 & 4607 & 281 & 6,7 & 4607 & 862 & 18,7 \\
\hline $1994 / 95$ & 33172 & 584 & 1,8 & 30294 & 3033 & 10,0 & 3327 & 229 & 6,9 & 3327 & 643 & 19,3 \\
\hline
\end{tabular}

Fonte: SUCEN

Entre as espécies triatomínicas presentes no estado, destaca-se T. sordida e P. megistus predominantemente peridomiciliares. No período analisado foram coletados 58.042 exemplares de triatomíneos, dos quais 36,7\% estavam presentes no intradomicílio (Tabela 4).

Tabela 4 - Triatomíneos coletados segundo espécie, local de captura e infecção natural. Estado de São Paulo, 1990 a 1995.

\begin{tabular}{|c|c|c|c|c|c|c|}
\hline \multirow[t]{2}{*}{ Espécie } & \multicolumn{2}{|c|}{ Intradomicílio } & \multicolumn{2}{|c|}{ Peridomicílio } & \multicolumn{2}{|c|}{ Total } \\
\hline & Col. & $\%^{*}$ & Col. & $\%^{*}$ & Col & $\% *$ \\
\hline Triatoma infestans & 2 & - & - & - & 2 & - \\
\hline Panstrongylus megistus & 5732 & 7,1 & 3275 & 8,3 & 9007 & 7,5 \\
\hline Triatoma sordida & 13741 & 0,2 & 33054 & 0,5 & 46795 & 0,4 \\
\hline Rhodnius neglectus & 1571 & 0,2 & 272 & 0,3 & 1843 & 0,2 \\
\hline Triatoma tibiamaculata & 364 & 15,3 & 31 & 16,1 & 395 & 15,4 \\
\hline Total & 21410 & 2,3 & 36632 & 1,2 & 58042 & 1,6 \\
\hline
\end{tabular}

Fonte: SUCEN

* \% infecção natural

Dentre os triatomíneos notificados, $82,1 \%$ estavam presentes no intradomicílio. Nos atendimentos à notificação a situação se inverteu contatando-se $80,1 \%$ de capturas no peridomicílio. Quanto a rotina, embora esta atividade tenha sido responsável pela coleta do maior número de triatomíneos (26.942), quando comparado as outras atividades, a maioria $(90,3 \%)$ estava no peridomicílio (Tabela 5).

Tabela 5 - Distribuição dos triatomíneos coletados segundo atividade e local de coleta. Estado de São Paulo, 1990 à 1995.

\begin{tabular}{|c|c|c|c|c|c|c|}
\hline \multirow{3}{*}{ Atividade } & \multicolumn{6}{|c|}{ Local de coleta } \\
\hline & \multicolumn{2}{|c|}{ intradomicílio } & \multicolumn{2}{|c|}{ peridomicílio } & \multicolumn{2}{|c|}{ total } \\
\hline & $\mathrm{n}^{0}$ & $\%$ & $\mathrm{n}^{0}$ & $\%$ & $\mathrm{n}^{0}$ & $\%$ \\
\hline$\overline{\text { Rotina }}$ & 2626 & 9,7 & 24316 & 90,3 & 26942 & 48,4 \\
\hline Notificação & 14319 & 82,1 & 3133 & 17,9 & 17452 & 30,1 \\
\hline Atendimento & 2729 & 19,9 & 10919 & 80,1 & 13648 & 23,5 \\
\hline$\overline{\text { Total }}$ & 19674 & 33,8 & 38368 & 66,2 & 58042 & $\overline{100,0}$ \\
\hline
\end{tabular}

Fonte: SUCEN

Quanto a colonização, o estudo dos triatomíneos coletados em atividade de atendimento à notificação, segundo espécie, mostrou que do total de exemplares de P. megistus, $19,3 \%$ consistia de ninfas presentes no intradomicílio e
$52,1 \%$ eram ninfas presentes no peridomicílio, enquanto que para $T$. sordida os percentuais foram de 4,8 e 41,9, respectivamente (Tabela 6).

Na Figura 1, se encontra a distribuição do T. sordida e $P$. megistus no biênio 94/95. 
Tabela 6 - Colonização do intadomicílio (ID) e peridomicílio $(P)$ segundo espécie triatomínica em coletas realizadas em atendimento à notificação. Estado de São Paulo, biênios 1990/91, 1992/93 e 1994/95.

\begin{tabular}{|c|c|c|c|c|c|c|c|c|c|c|c|c|c|c|c|}
\hline \multirow{3}{*}{ Biênio } & \multicolumn{5}{|c|}{ P. megistus } & \multicolumn{5}{|c|}{ T. sordida } & \multicolumn{5}{|c|}{ R. neglectus } \\
\hline & \multirow[b]{2}{*}{ total col. } & \multicolumn{4}{|c|}{ ninfa } & \multirow[b]{2}{*}{ total col. } & \multicolumn{4}{|c|}{ ninfa } & \multirow[b]{2}{*}{ total col. } & \multicolumn{4}{|c|}{ ninfa } \\
\hline & & ID & $\%$ & $\mathrm{P}$ & $\%$ & & ID & $\%$ & $P$ & $\%$ & & ID & $\%$ & $\mathrm{P}$ & $\%$ \\
\hline $1990 / 91$ & 982 & 139 & 14,1 & 647 & 65,9 & 3753 & 279 & 7,4 & 2108 & 56,2 & 59 & 2 & 3,4 & 10 & 16,9 \\
\hline $1992 / 93$ & 1039 & 291 & 28,0 & 491 & 47,2 & 2582 & 234 & 9,1 & 1225 & 47,4 & 23 & - & - & 12 & 52,2 \\
\hline $1994 / 95$ & 670 & 91 & 13,6 & 266 & 39,7 & 6874 & 123 & 1,8 & 2211 & 32,2 & 84 & 15 & 17,8 & 28 & 33,3 \\
\hline Total & 2691 & 521 & 19,3 & 1404 & 52,1 & 13209 & 636 & 4,8 & 5544 & 41,9 & 166 & 17 & 10,2 & 50 & 30,1 \\
\hline
\end{tabular}

Fonte: SUCEN

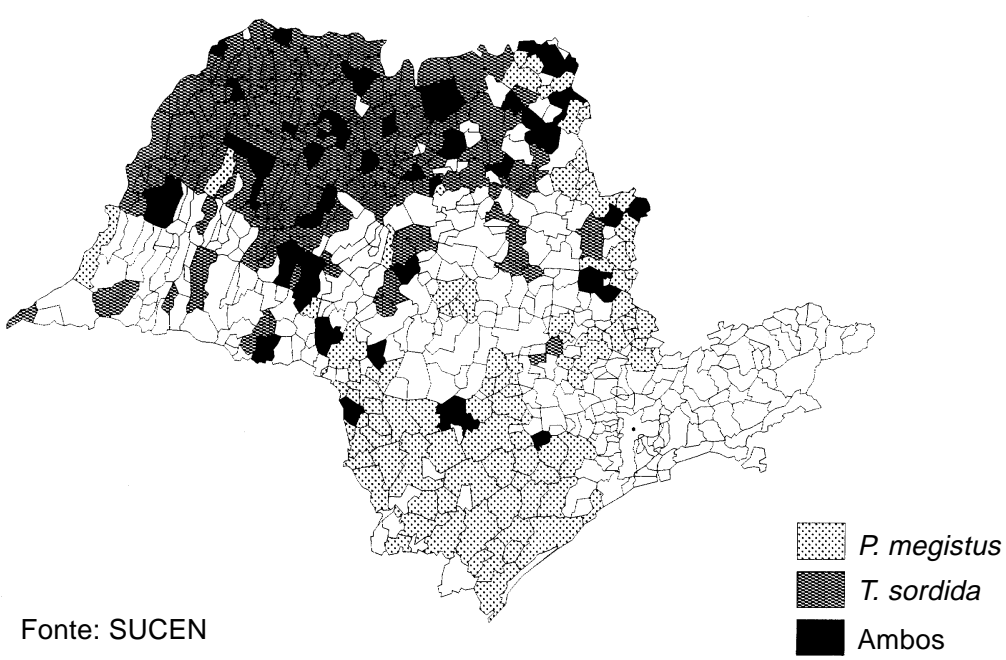

Figura 1 - Distribuição de Triatoma sordida e Panstrongylus megistus no Estado de São Paulo, biênio 94/95.

\section{DISCUSSÃO}

O controle da doença de Chagas no Estado de São Paulo está centrado no combate aos vetores domiciliados cujas atividades são realizadas pela SUCEN. O Estado apresenta hoje um espaço bastante reduzido de concentração triatomínica no ambiente domiciliar, restrito a poucas localidades, não mais se justificando pesquisas rotineiras casa-a-casa, em toda a área rural, por parte do serviço de controle. A revisão do Programa realizada em 1990 confirmou como encerrada a campanha contra o Triatoma infestans no Estado, entretanto, no sentido de avaliar uma possível reintrodução da espécie, as atividades de vigilância foram mantidas, juntamente com o controle dirigido aos demais triatomíneos invasores dos ecótopos artificiais ${ }^{7}$. Assim, muito embora vários estudos já tivessem apontado no sentido da suspensão das ações de busca ativa de triatomíneos por parte do Serviço de controle ${ }^{236}$, priorizando uma fase da vigilância entomológica com a participação popular, a metodologia definida pelo Programa recomendou pesquisa triatomínica em área de infestação residual ${ }^{9}$. De acordo com Dias ${ }^{4}$, intervenções verticais realizadas anualmente são onerosas e pouco sensíveis na detecção de triatomíneos, especialmente em casas com baixa densidade de vetores. Também mostraram baixa capacidade de detecção de infestação, quando comparadas com a notificação em estudo de métodos alternativos de vigilância de triatomíneos 
em área endêmica de Goiás ${ }^{13}$. As análises recentes das informações coletadas no Programa de Controle no Estado de São Paulo tem apontado que a participação da população na notificação triatomínica se mostra de grande eficácia na descoberta de focos de triatomíneos, uma vez que a notificação constitui seleção prévia de casas a serem pesquisadas e, portanto, com maior chance de serem encontradas infestadas ${ }^{10} 1112$.

A notificação de triatomíneos por parte da população passou a ser incentivada pelas equipes de campo da SUCEN em suas atividades rotineiras de pesquisa nos domicílios rurais, a partir da implantação da fase de vigilância entomológica, nos anos 70, quando os indicadores soroepidemiológicos e entomológicos apresentavam que a transmissão vetorial da doença de Chagas por Triatoma infestansestava sob controle. A partir de 1983 foi introduzida a priorização da pesquisa domiciliar, em resposta a uma notificação de triatomíneo encaminhada por moradores, sobre a programação de rotina. As atividades educativas passaram a incluir o treinamento de equipes para que pudessem fornecer à população informações necessárias a compreensão da importância do trabalho de pesquisa de triatomíneos, solicitando quando possível a presença do morador no decorrer da pesquisa, orientando-o quanto aos locais mais habituais de encontro dos barbeiros ${ }^{12}$.

Ao mesmo tempo se buscou trabalhar junto as escolas rurais, sendo a professora utilizada como elemento central, motivando e capacitando a população para o desempenho da função de notificante. Esta estratégia foi implementada por Dias e Garcia $^{2}$ no município de Bambuí e demonstrou que a população responde de forma efetiva a ações de seus interesses na medida que compreendem os processos e os problemas que lhe afetem de modo direto ${ }^{5}$.

O processo de detecção de triatomíneos com a participação da população significa uma vigilância contínua se comparado as atividades rotineiras realizadas pelas equipes de campo. $\mathrm{Na}$ fase da vigilância entomológica a cooperação da população é eficaz pois, sensibilizado, o morador se mantém constantemente atento ao surgimento de barbeiros ${ }^{3}$. Nesse quadro, também se acentua a redução de triatomíneos domiciliados, inclusive no peridomicílio onde se concentra a grande maioria dos focos residuais. No peridomicílio os inseticidas não funcionam bem, daí requerendose a imprescindível participação dos moradores na manutenção deste ambiente organizado e limpo com atenção especial para locais que ofereçam abrigo e alimento para triatomíneos ${ }^{5}$.

A pesquisa minuciosa nas unidades que notificaram o encontro de triatomíneos nas casas detectou a presença de focos em $26,2 \%$ delas (Tabela 1). Adiminuição do número de notificações ao longo do período, pode sinalizar para um descuido por parte do Serviço em não priorizar $o$ atendimento, desestimulando a população. No entanto, esse aspecto deve ser melhor avaliado uma vez que o aumento dos índices de infestação obtidos na rotina (intra e peridomicílio) e atendimento à notificação (peridomicílio) pode ser caracterizado pela manutenção dos focos no peridomicílio, e invasão do intradomicílio por fêmeas visitantes, daí, a possibilidade de instalação de focos no intradomicílio. Note-se, porém, que os dados aqui analisados demonstraram não haver aumento na infestação do intradomicílio no atendimento à notificação.

As notificações procederam de localidades independentemente dos seus níveis de infestação. Este aspecto é relevante, principalmente ao se considerar as localidades classificadas como P3 que, teoricamente livres de infestação, demonstraram percentuais médios de positividade nos atendimentos em torno de $26,0 \%$, o que as coloca em posição importante no sistema de vigilância ${ }^{12}$ (Tabela 2 ).

Os resultados das pesquisas triatomínicas realizadas nas atividades de atendimento à notificação quando comparadas com os obtidos nas atividades de rotina, demonstram claramente a importância da participação da população na vigilância (Tabela 3). Os atendimentos à notificação têm mostrado percentuais de positividade mais elevados tanto para casas como para peridomicílios se comparados a rotina ${ }^{12}$.

Verificou-se que $82,1 \%$ dos exemplares coletados no intradomicílio (Tabela 4), estiveram condicionados à notificação pelo morador e que a pesquisa posterior nestas residências detectou mais $19,1 \%$ dos triatomíneos, deixa claro a importância da notificação na vigilância do intradomicílio e, consequentemente, na vigilância do risco de transmissão da doença de Chagas por esses vetores. Ao contrário, a rotina está relacionada a vigilância do peridomicílio (Tabela 5). A colonização intradomiciliar constitui fator básico na transmissão da doença de Chagas ao homem. O encontro de ninfas no intradomicílio, embora associado a foco peridomiciliar se deve a invasão de fêmeas férteis encontradas pelos moradores 
e posteriormente notificadas, dando-se a eclosão dos ovos e a permanência das ninfas na casa que são coletadas quando do atendimento à notificação pelas equipes de campo (Tabela 6). Estas constatações têm orientado as recentes reformulações das ações de vigilância entomológica da doença de Chagas no Estado de São Paulo ${ }^{11} 12$.

$\mathrm{Na}$ fase atual em que se encontra o PCDCh no Estado de São Paulo, é necessário a busca de mecanismos alternativos que propiciem efetivamente um grande envolvimento da população na vigilância triatomínica. Na vigilância vetorial da doença de Chagas no Estado de São Paulo, as intervenções verticais que, além de consumir os já escassos recursos existentes, se mostraram menos eficazes do que as notificações de triatomíneos pela população e poderiam ser eliminadas da proposta metodológica do Programa, a exemplo do que já vem sendo implementado em outras áreas do Brasil e de outros países ${ }^{1}$.

\section{AGRADECIMENTO}

Ao Prof. Dr. Fernando M.A. Corrêa pela revisão crítica do manuscrito.

\section{REFERÊNCIAS BIBLIOGRÁFICAS}

1. Bryan RT, Balderrama F, Tonn RJ, Dias JCP. Community participation in vector control: Lessons from Chagas disease. The American Journal of Tropical Medicine and Hygiene 50(suppl):61-71, 1994.

2. Dias JCP, Garcia ALR. Vigilância epidemiologica con participacion comunitaria. Un programa de enfermedad de Chagas. Boletin de la Oficina Sanitaria Panamericana 84: 533-544, 1978.

3. Dias JCP, Dias RB. Participação da comunidade no controle da doença de Chagas. Anales de la Societè Belge de Medicine Tropicale 65(supl 1):127-135, 1985.

4. Dias JCP. Chagas' disease control in Brazil: Which strategy after the attack phase? Annales Societe Belge Medicine Tropicale 71(suppl 1):75-86, 1991.

5. Dias JCP. Problemas e possibilidades de participação comunitária no controle das grandes endemias no Brasil. Cadernos de Saúde Pública 14(supl 2):19-37, 1998.

6. Rocha e Silva EO, Maluf J, Corrêa RR. Doença de Chagas: atividades de vigilância entomológica numa área do Estado de São Paulo. Revista de Saúde Pública 4:129145,1970.

7. Rocha e Silva EO, Wanderley DMV, Rodrigues VLCC. Triatoma infestans: importância, controle e eliminação da espécie no Estado de São Paulo, Brasil. Revista da
Sociedade Brasileira de Medicina Tropical 31:73-88, 1998.

8. Silva EOR, Guarita OF, Ishihata GK. Doença de Chagas: Atividades de controle dos transmissores no Estado de São Paulo. Revista Brasileira de Malariologia 31:99-119, 1979.

9. Secretaria de Estado da Saúde - Superintendência de Controle de Endemias - Relatório Final do Grupo de Trabalho do Programa de Controle da Doença de Chagas no Estado de São Paulo 1989.

10. Souza AG, Wanderley DMV, Buralli GM, Andrade JCR. Consolidation of the control of Chagas' disease vectors in the State of São Paulo. Memórias do Instituto Oswaldo Cruz, 79:125-31, 1984.

11. Wanderley DMV. Vigilância Epidemiológica da doença de Chagas no Estado de São Paulo. Revista Saúde Pública 25:28-32, 1991.

12. Wanderley DMV. Perspectivas de Controle da doença de Chagas no Estado de São Paulo. Tese de Doutorado, Faculdade de Saúde Pública da Universidade de São Paulo, São Paulo, 1994.

13. Zapata MTG, Marsden P. Enfermedad de Chagas: control y vigilância con inseticidas y participacion comunitária em Mambai, Goiás. Brasil. Boletín de la Oficina Sanitaria Panamericana 116:97-110,1994. 